\begin{tabular}{|c|c|}
\hline Citation & $\begin{array}{l}\text { Dora Turk, Taranjitsingh Singh and Jan Swevers (2018). } \\
\text { Linear parameter-varying system identification of an } \\
\text { industrial ball screw setup. } \\
\text { The 15th International Workshop on Advanced Motion Control (AMC2018). }\end{array}$ \\
\hline Archived version & $\begin{array}{l}\text { Author manuscript: the content is identical to the content of the published } \\
\text { paper, but without the final typesetting by the publisher }\end{array}$ \\
\hline Published version & \\
\hline Journal homepage & http://ieeexplore.ieee.org/xpl/conhome.jsp?punumber $=1000012$ \\
\hline Author contact & $\begin{array}{l}\text { dora.turk@kuleuven.be } \\
+32(0) 16372857\end{array}$ \\
\hline IR & https://lirias.kuleuven.be/handle/123456789/606900 \\
\hline
\end{tabular}

(article begins on next page) 


\title{
Linear parameter-varying system identification of an industrial ball screw setup
}

\author{
Dora Turk, Taranjitsingh Singh and Jan Swevers \\ MECO Research Team, Department of Mechanical Engineering, KU Leuven, Belgium \\ DMMS lab, Flanders Make, Leuven, Belgium \\ Email: firstname.lastname@kuleuven.be
}

\begin{abstract}
Ball screws are high-precision positioning systems that translate rotational motion of an electric motor into translational motion of a slide. Due to the distributed stiffness and inertia of the ball screw, the resonance frequencies of the system vary with the position of the slide. This paper discusses the experimental identification of an industrial ball screw setup by treating it as a linear parameter-varying (LPV) system. Application of a novel nonlinear least-squares LPV system identification method with $\ell_{2,1}$-norm regularization yields a sparse model with satisfactory accuracy both in time and frequency domain and over the whole operating range of the setup.
\end{abstract}

Index Terms-ball screw drive, linear parameter-varying systems, system identification.

\section{INTRODUCTION}

Ball screws are high-precision positioning systems frequently used in industrial automation and robotics. Ball screw drives translate rotational motion of an electric motor into translational motion of a nut with attached slide. The slide thus performs a desired motion along the ball screw shaft while carrying a load or a workpiece. An example of such a system is shown in Fig. 1.

The frequency responses of ball screws disclose torsional and translational resonances, that happen to depend on the mass of the load mounted on the slide, and on the actual position of the slide along the shaft, as a consequence of the distributed stiffness and inertia of the shaft. Henke et al. [1] give a concise overview of available modeling techniques for industrial-scale ball screw motion systems and present a detailed modeling and experimental validation of a particular ball screw setup based on Ritz series discretization.

This paper discusses the experimental identification of the industrial ball screw setup shown in Fig. 1 by treating it as a linear parameter-varying (LPV) system. LPV systems are nonlinear systems described by a linear model with coefficients varying as a function of one or more scheduling parameters. The resulting model captures the dominant resonance and antiresonance frequency as a function of the position of the slide, which is both the scheduling parameter and a model output.

The literature on LPV system identification distinguishes between global and local approaches. The global techniques

This work has been carried out within the framework of Flanders Make SBO ROCSIS project (Robust and Optimal Control of Systems of Interacting Subsystems), FWO G.0915.14 project of the Research Foundation - Flanders (FWO - Flanders), and KU Leuven-BOF PFV/10/002 Centre of Excellence: Optimization in Engineering (OPTEC).

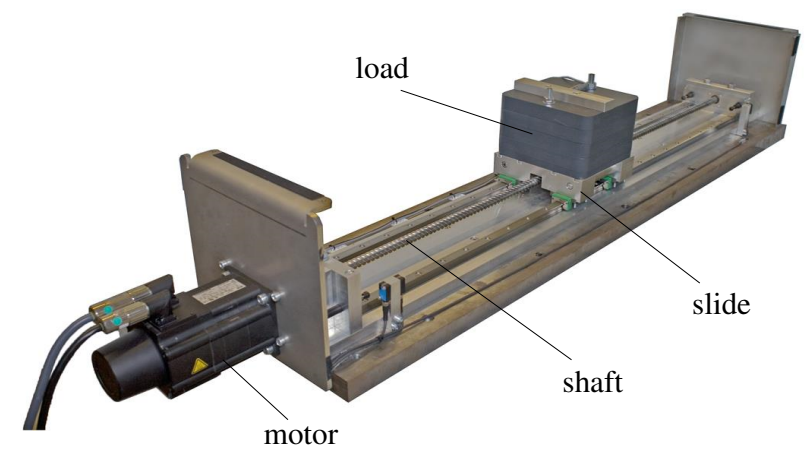

Fig. 1: The industrial ball screw setup on which the experimental LPV model identification has been performed.

(e.g. [2]) directly identify an LPV model based on data obtained from experiments where both the input signal and the scheduling parameters are continuously changing. Experiments of this kind are referred to as global experiments. Local identification techniques (e.g. [3]) identify an LPV model based on data obtained from several local experiments during which the scheduling parameters are fixed.

Aside the type of the experiments, an important query in LPV system identification is the determination of an adequate dependency of the model on the scheduling parameter(s). Keeping in mind that a complex dependency complicates consequent LPV control synthesis and analysis, this paper applies a novel nonlinear least-squares local LPV frequency domain system identification method with $\ell_{2,1}$-norm regularization [4]. The method directly identifies state-space models. Through a reweighted $\ell_{2,1}$-norm regularization, an automatic reduction of model structure complexity is accomplished by discarding redundant basis function dependencies from the state-space matrices.

The paper is organized as follows. Section II introduces the chosen LPV model structure, the identification data and the applied identification method proposed in [4]. Section III discusses in detail the experimental identification of the ball screw setup and analyzes the obtained model sparsity and performance, in the local and global sense. Section IV brings the conclusions. 


\section{LPV SYSTEM IDENTIFICATION}

\section{A. Model structure}

In this paper we focus on the following fully parameterized discrete-time state-space model:

$$
\left\{\begin{array}{l}
x(t+1)=\mathscr{A}(p(t)) x(t)+\mathscr{B}(p(t)) u(t) \\
y(t)=\mathscr{C}(p(t)) x(t)+\mathscr{D}(p(t)) u(t),
\end{array}\right.
$$

where $x(t) \in \mathbb{R}^{n}, u(t) \in \mathbb{R}^{r}, y(t) \in \mathbb{R}^{l}$ and $p(t) \in \mathbb{R}^{N_{p}}$ are, respectively, the state vector, the input vector, the output vector, and the scheduling parameter vector, at time instance $t$. The state-space matrices of the introduced model are parameterdependent:

$$
\mathscr{X}(p(t))=X^{(0)}+\sum_{i=1}^{N_{b}} X^{(i)} \psi_{i}(p(t))
$$

$\forall(\mathscr{X}, X) \in\{(\mathscr{A}, A),(\mathscr{B}, B),(\mathscr{C}, C),(\mathscr{D}, D)\} ; A^{(i)} \in \mathbb{R}^{n \times n}$ $B^{(i)} \in \mathbb{R}^{n \times r}, C^{(i)} \in \mathbb{R}^{l \times n}, D^{(i)} \in \mathbb{R}^{l \times r}, \forall i=0,1, \ldots, N_{b}$, where $N_{b}$ is the number of basis functions $\psi_{i}$ of the scheduling parameter $p(t)$ that are employed for the parameterization. No particular choice of basis functions is here assumed. Since only local identification data are considered in this paper, the basis functions $\psi_{i}$ are considered to depend only on the instantaneous time values of the scheduling parameter $p(t)$, which excludes dynamic scheduling parameter dependency. For simplicity of notation, all model matrices $\{\mathscr{A}, \mathscr{B}, \mathscr{C}, \mathscr{D}\}$ are assumed to have the same number of basis functions. This assumption can, however, be relaxed without loss of generality. The model parameters are stacked into a vector $\theta$ in the following way:

$$
\begin{gathered}
\theta_{X^{(i)}}=\operatorname{vec}\left(X^{(i)}\right), \quad \forall X \in\{A, B, C, D\}, \quad \forall i=0, \ldots, N_{b}, \\
\theta_{X}=\left[\begin{array}{lllll}
\theta_{X^{(0)}}^{T} & \theta_{X^{(1)}}^{T} & \ldots & \theta_{X^{\left(N_{b}\right)}}^{T}
\end{array}\right]^{T}, \quad \forall X \in\{A, B, C, D\}, \\
\theta=\left[\begin{array}{llll}
\theta_{A}^{T} & \theta_{B}^{T} & \theta_{C}^{T} & \theta_{D}^{T}
\end{array}\right]^{T} .
\end{gathered}
$$

\section{B. Identification data}

The identification data set assumed in this paper consists of frequency response function (FRF) matrices of the system measured at a specified set of frequencies $\omega_{k}$, and for a specified set of fixed values of the scheduling parameter $p^{[q]}$, that is:

$$
\left\{G_{\mathrm{m}}^{[q]}\left(\omega_{k}\right)\right\}, k=1, \ldots, F^{[q]}, q=1, \ldots, N .
$$

The measured FRFs $\left(G_{\mathrm{m}}^{[q]}\left(\omega_{k}\right)\right)$ contain stochastic components (originating from e.g. measurement noise) that can be present on both input and output, internal system disturbances and nonlinearities.

\section{A reweighted $\ell_{2,1}$-norm regularization approach}

In parameter estimation, a commonly used fitness criterion is the squared error:

$$
V_{\mathrm{NLS}}(\theta)=\varepsilon(\theta)^{\mathrm{H}} \varepsilon(\theta),
$$

where $\varepsilon(\theta)$ denotes the difference between the model response and the corresponding measured system response, and $\varepsilon(\theta)^{\mathrm{H}}$ is the conjugate transpose of $\varepsilon(\theta)$. The frequency domain model error vector for each $q=1, \ldots, N$ equals

$$
\left.\varepsilon^{[q]}(\theta)=\left[\begin{array}{llll}
\varepsilon^{[q]}\left(\theta, \omega_{1}\right)^{T} & \varepsilon^{[q]}\left(\theta, \omega_{2}\right)^{T} & \ldots & \varepsilon^{[q]}\left(\theta, \omega_{F}[q]\right.
\end{array}\right)^{T}\right]^{T}
$$

and consists of:

$$
\varepsilon^{[q]}\left(\theta, \omega_{k}\right)=\operatorname{vec}\left(G^{[q]}\left(\theta, \omega_{k}\right)-G_{\mathrm{m}}^{[q]}\left(\omega_{k}\right)\right), \quad k=1, \ldots, F^{[q]},
$$

where $G^{[q]}\left(\theta, \omega_{k}\right)$ is the model frequency response function matrix. Eventually, all data are stacked in one vector, i.e.:

$$
\varepsilon(\theta)=\left[\begin{array}{llll}
\varepsilon^{[1]}(\theta)^{T} & \varepsilon^{[2]}(\theta)^{T} & \ldots & \varepsilon^{[N]}(\theta)^{T}
\end{array}\right]^{T} .
$$

The nonlinear least-squares parameter estimate is given by

$$
\hat{\theta}=\arg \min _{\theta} V_{\mathrm{NLS}}
$$

and can be calculated via the Levenberg-Marquardt algorithm. As mentioned in the introduction, to select the most appropriate scheduling parameter dependency, this paper follows a regularization approach [4]. The approach assumes a predefined set of basis functions-based on physical insights or guess-to describe the scheduling parameter dependency of the initial, purposely too complex model. Throughout the identification algorithm, the model complexity is iteratively reduced by discarding basis function dependencies from the state-space matrices where they are assessed as redundant. This strategy is expressed through the following nonlinear second-order cone programming (NSOCP) problem:

$$
\begin{array}{r}
\underset{\theta, s}{\operatorname{minimize}} V_{\mathrm{NLS}}(\theta)+\gamma \sum_{i=1}^{N_{b}}\left(\phi\left(\hat{\theta}_{A^{(i)}}\right) s_{A^{(i)}}+\phi\left(\hat{\theta}_{B^{(i)}}\right) s_{B^{(i)}}+\right. \\
\left.+\phi\left(\hat{\theta}_{C^{(i)}}\right) s_{C^{(i)}}+\phi\left(\hat{\theta}_{D^{(i)}}\right) s_{D^{(i)}}\right), \\
\text { subject to } \varphi\left(\hat{\theta}_{A^{(i)}}\right)\left\|\theta_{A^{(i)}}\right\|_{2} \leq s_{A^{(i)}}, \\
\varphi\left(\hat{\theta}_{B^{(i)}}\right)\left\|\theta_{B^{(i)}}\right\|_{2} \leq s_{B^{(i)}}, \\
\varphi\left(\hat{\theta}_{C^{(i)}}\right)\left\|\theta_{C^{(i)}}\right\|_{2} \leq s_{C^{(i)}}, \\
\varphi\left(\hat{\theta}_{D^{(i)}}\right)\left\|\theta_{D^{(i)}}\right\|_{2} \leq s_{D^{(i)}}, \\
i=1, \ldots, N_{b} .
\end{array}
$$

In (12), $\gamma$ is a scalar, of which the value determines the rigorousness of the penalization, that is, the importance of the model simplicity with regard to the accuracy $V_{\mathrm{NLS}}(\theta)$. The penalization weights $\phi$ are selected such that model parameters not contributing substantially to the system response are penalized, more precisely:

$$
\phi\left(\hat{\theta}_{X^{(i)}}\right)=\left(\left\|\hat{\theta}_{X^{(i)}}\right\|_{2}\left\|\psi_{i}\right\|_{\infty}+\varepsilon\right)^{-1},
$$

$\forall X \in\{A, B, C, D\}, \forall i=1, \ldots, N_{b} . \hat{\theta}_{X^{(i)}}$ denotes an estimate of the parameter vector $\theta_{X^{(i)}}$ available prior to the solution of (12), and $\left\|\psi_{i}\right\|_{\infty}$ is the maximal absolute value of $\psi_{i}$. A small tuning parameter $\varepsilon$ is added for numerical stability and by tuning it, one tunes the intensity of the penalization strategy. 
A step further in assessing the importance of a particular subset of model parameters is comparing their contribution to a statespace matrix against the LTI contribution, which yields:

$$
\phi\left(\hat{\theta}_{X^{(i)}}\right)=\left(\frac{\left\|\hat{\theta}_{X^{(i)}}\right\|_{2}\left\|\psi_{i}\right\|_{\infty}}{\left\|\hat{\theta}_{X^{(0)}}\right\|_{2}}+\varepsilon\right)^{-1}
$$

$\forall X \in\{A, B, C, D\}, \forall i=1, \ldots, N_{b}$. With additional weights $\varphi$

$$
\varphi\left(\hat{\theta}_{X^{(i)}}\right)=\frac{\left\|\psi_{i}\right\|_{\infty}}{\left\|\hat{\theta}_{X^{(0)}}\right\|_{2}},
$$

the value of the regularization part of the optimization criterion will converge to the number of nonzero subsets $\theta_{X^{(i)}}$, namely

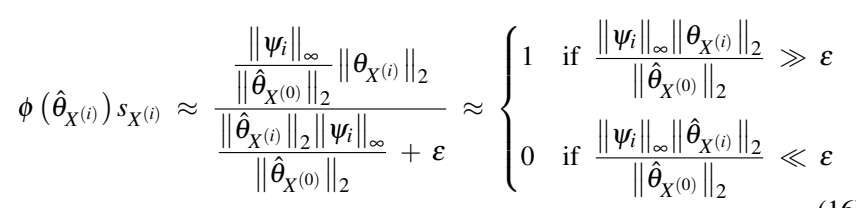

$\forall X \in\{A, B, C, D\}, \forall i=1, \ldots, N_{b}$, which is convenient for keeping track of the algorithm progress. In case there exist $X$ for which $\left\|\hat{\theta}_{X^{(0)}}\right\|_{2}=0$, expression (13) should be used instead. Motivated by the Levenberg-Marquardt algorithm, we solve (12) by "cutting" it into convex SOCP subproblems. The described principle makes sense only under assumption that the weights are based upon a valid set of parameter estimates $\hat{\theta}$. The algorithm, therefore, alternates between estimating $\theta$ by solving (12) and redefining the penalization weights, as in [5]. Details on its implementation can be found in [4].

\section{EXPERIMENTAL VALIDATION}

This section discusses the application of the described LPV identification approach to the industrial ball screw system shown in Fig. 1. The system translates rotational motion of a $1.1 \mathrm{~kW}$ Lenze MCS $12 \mathrm{D} 20$ synchronous servo motor into translational motion of a slide. The setup is equipped with a linear encoder attached to the slide with a resolution of $0.5 \mu \mathrm{m}$, and a rotary encoder giving 4096 pulses per revolution yielding, with a ball screw pitch of $50 \mathrm{~mm}$, a resolution of about $12 \mu \mathrm{m}$. The torque constant equals $2.34 \mathrm{Nm} / \mathrm{A}$. The slide is loaded with an additional mass of $45 \mathrm{~kg}$, yielding a total mass of $51 \mathrm{~kg}$. An xPC Target (Speedgoat) machine ensures real-time operation at a sampling rate of $4 \mathrm{kHz}$.

Due to the distributed stiffness and inertia of the flexible ball screw, the resonance frequencies of the system vary with the slide position [1]. The slide's position is, thus, the scheduling parameter of the system we aim to identify. For this setup, we opted for closed-loop identification, that is, identification of the open-loop system dynamics in the presence of a feedback controller. Direct open-loop identification turned out to be impractical because of system drift due to system nonlinearities (cogging and actuator torque offset) continuously disturbing the measurements and not allowing periodicity. Fig. 2 shows the applied closed-loop measurement configuration. The system has two outputs, motor angular position $y_{1}$ and

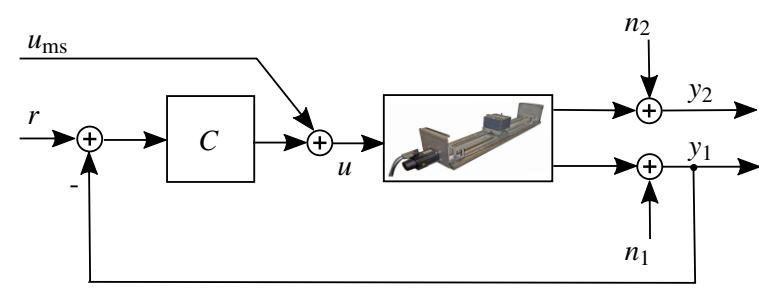

Fig. 2: Closed-loop measurement configuration

the slide position $y_{2}$. The feedback controller, schematically represented in Fig. 2 by $C$, is a cascaded proportional-integral velocity and propotional position feedback controller, resulting in a closed-loop bandwidth of $2.5 \mathrm{~Hz}$. A low bandwith is selected for this controller as its sole purpose is to prevent the system from drifting away from the desired operating point $r$, while it is being excited by a signal $u_{\mathrm{ms}}$. As a result of this low bandwith, the interference of the controller with the excitation signal $u_{\mathrm{ms}}$ around the system's anti-resonance and resonance frequencies is low. The excitation signal $u_{\mathrm{ms}}$ and the scheduling reference $r$ are noise-free, whereas the system outputs $y_{i}, i=1,2$ are corrupted with stochastic disturbances $n_{i}$ comprising of process and measurement noise, and nonlinear distortions (coming from e.g. nonlinear friction, backlash and cogging).

In this closed-loop setting the system input $u$ is corrupted with stochatic disturbances that are correlated with the (process and measurement) noise on the output that is fed back. Because of this correlation, the calculation of the ball screw FRFs relating input $u$ to outputs $y_{1}$ and $y_{2}$ is intricate. A calculation of these FRFs directly from the measurement of these signals introduces bias. To avoid this bias, the so-called indirect method is applied [6] (section 7.2.7), that is:

$$
G_{\mathrm{m}}^{[q]}\left(\omega_{k}\right)=G_{u y_{i}}^{[q]}\left(\omega_{k}\right)=\frac{G_{u_{\mathrm{ms}} y_{i}}^{[q]}\left(\omega_{k}\right)}{G_{u_{\mathrm{ms}} u}^{q q]}\left(\omega_{k}\right)},
$$

$i=1,2, k=1, \ldots, F^{[q]}, q=1, \ldots, N$, where $G_{u_{\mathrm{ms}} y_{i}}^{[q]}$ and $G_{u_{\mathrm{ms}} u}^{[q]}$ are frequency response functions estimated in the, typically open-loop, setting with noise-free input $u_{\mathrm{ms}}$ and noisy outputs $y_{i}$ or $u$, which means that the robust FRF estimation method found in [6] (section 4.3.1) can be applied to obtain consistent FRF estimates. The resulting $G_{u y_{i}}^{[q]}$ is then also consistent.

\section{A. Experiment design}

Given the operating range of the setup, the following positions of the slide were chosen as scheduling points at which local experiments were performed:

$$
p=\{-0.35,-0.1625,-0.0688,0.025,0.2125,0.4\} \mathrm{m},
$$

where $p=-0.35 \mathrm{~m}$ corresponds to the position near the motor, and $p=0.4 \mathrm{~m}$ to the far end of the ball screw. The measurement taken at the position $p=-0.0688 \mathrm{~m}$ is used for validation purposes only, whereas the remaining five are used for the identification. At each position, four experiments with different realizations of a random-phase multisine signal 
were performed. During each experiment, fifteen periods of the steady-state response were measured. By performing several experiments each with a different realization of a randomphase multisine, and by measuring several periods during each experiment, it is possible to estimate the total sample covariance matrices and the sample noise covariance matrices of the FRF estimates $G_{u_{\mathrm{ms}} u}$ and $G_{u_{\mathrm{ms}} y_{i}}, \forall i=1,2$, [6] (section 4.3.1). The total sample covariance matrices are a measure of the stochastic nonlinear distortions and measurement and process noise. The sample noise covariance matrices are a measure of the measurement and process noise only. The diagonal elements of these matrices are the estimates of the variances, whereas the off-diagonal elements describe the correlation between the distortions and noise on both FRF estimates, correlation introduced by the feedback controller. Based on these covariance matrix estimates, the total sample variance and noise sample variance of $G_{u y_{i}}, \forall i=1,2$ can be calculated as in [6], section 7.2.7.1, expression (7-50). These variances are used later in this paper to evaluate the model accuracy. The multisine signals are composed of frequencies in the range $f \in[1,150] \mathrm{Hz}$, with an as high as possible amplitude without causing motor current saturation when combined with the controller action, and a duration of $4.096 \mathrm{~s}$ resulting in a frequency resolution of $0.2441 \mathrm{~Hz}$.

\section{B. Model structure selection}

Just like any nonlinear least-squares approach, the applied identification approach requires estimates of the model parameters to start from. These initial estimates $\theta_{0}$ are here generated using the State-space Model Interpolation of Local Estimates (SMILE) technique [3]. The SMILE technique starts from LTI models estimated via the nonlinear least-squares frequency domain system identification method [6] based on the aforementioned FRFs. Since we know there is an integrator in the system, i.e. a pole at $z=1$, this prior knowledge is used to simplify the identification. Namely, the integral action is removed from the FRFs via multiplication by $\left(e^{j \omega T_{s}}-1\right) / T_{s}$, with $T_{s}$ being the sampling time, and will be added back to the state-space model obtained after the LPV identification. The identified LTI models are of order three. The SMILE technique interpolates the LTI models using basis functions of the scheduling parameter. Since there is no prior knowledge available on the scheduling parameter dependency of the model, and since there are five LTI models, a fourthorder polynomial scheduling parameter dependency and hence following set of basis functions:

$$
\psi_{1}=p(t), \quad \psi_{2}=p(t)^{2}, \quad \psi_{3}=p(t)^{3}, \quad \psi_{4}=p(t)^{4},
$$

was chosen. With this choice, the interpolation can be performed without introducing errors; the LTI models match exactly to the LPV model for the corresponding fixed values of the scheduling parameter. Tables I-II show for the obtained SMILE model the magnitude of the parameters of the matrices linked to the different basis functions; Table I displays their $\ell_{2}$ norm, whereas Table II shows their relative magnitude indicating the contribution to the system response compared to the linear counterpart. It can be seen that all basis functions $\left(\psi_{1}, \ldots, \psi_{4}\right)$ are being used in each model matrix $(\mathscr{A}, \mathscr{B}, \mathscr{C}, \mathscr{D})$. To reduce the complexity of the scheduling parameter dependency of the LPV model, the reweighted $\ell_{2,1^{-}}$ norm regularization approach (section II-C) is applied, and the results are summarized in Table III and Table IV.

TABLE I: Parameter magnitude of the SMILE model: $\ell_{2,1}$-norm elements

\begin{tabular}{|c|c|c|c|c|}
\hline $\mathbf{i}$ & $\left\|\theta_{A^{(i)}}\right\|_{2}$ & $\left\|\theta_{B^{(i)}}\right\|_{2}$ & $\left\|\theta_{C^{(i)}}\right\|_{2}$ & $\left\|\theta_{D^{(i)}}\right\|_{2}$ \\
\hline 0 & 1.0066 & 0.0470 & 0.0401 & 0.0009 \\
\hline 1 & 0.1043 & 0.0063 & 0.0001 & 0.0008 \\
\hline 2 & 0.0850 & 0.0909 & 0.0017 & 0.0100 \\
\hline 3 & 0.1866 & 0.1213 & 0.0023 & 0.0077 \\
\hline 4 & 0.4676 & 0.4900 & 0.0064 & 0.0542 \\
\hline
\end{tabular}

TABLE II: Parameter magnitude of the SMILE model: weighted $\ell_{2,1}$-norm elements

\begin{tabular}{|c|c|c|c|c|}
\hline $\mathbf{i}$ & $\frac{\left\|\psi_{i}\right\|\left\|_{\infty}\right\| \theta_{A^{(i)}} \|_{2}}{\left\|\hat{\theta}_{A^{(0)}}\right\|_{2}}$ & $\frac{\left\|\psi_{i}\right\|_{\infty}\left\|\theta_{B^{(i)}}\right\|_{2}}{\left\|\hat{\theta}_{B^{(0)}}\right\|_{2}}$ & $\frac{\left\|\psi_{i}\right\|_{\infty}\left\|\theta_{C^{(i)}}\right\|_{2}}{\left\|\hat{\theta}_{C^{(0)}}\right\|_{2}}$ & $\frac{\left\|\psi_{i}\right\|_{\infty}\left\|\theta_{D^{(i)}}\right\|_{2}}{\left\|\hat{\theta}_{D^{(0)}}\right\|_{2}}$ \\
\hline 1 & 0.0242 & 0.0539 & 0.0010 & 0.3813 \\
\hline 2 & 0.0079 & 0.3095 & 0.0058 & 1.8417 \\
\hline 3 & 0.0069 & 0.1653 & 0.0030 & 0.5689 \\
\hline 4 & 0.0070 & 0.2670 & 0.0035 & 1.5978 \\
\hline
\end{tabular}

\section{Identification results}

TABLE III: Parameter magnitude of the regularized model: $\ell_{2,1}$-norm elements

\begin{tabular}{|c|c|c|c|c|}
\hline $\mathbf{i}$ & $\left\|\theta_{A^{(i)}}\right\|_{2}$ & $\left\|\theta_{B^{(i)}}\right\|_{2}$ & $\left\|\theta_{C^{(i)}}\right\|_{2}$ & $\left\|\theta_{D^{(i)}}\right\|_{2}$ \\
\hline 0 & 1.0070 & 0.0412 & 0.0488 & 0.0007 \\
\hline 1 & 0.1041 & $8.5254 \cdot 10^{-16}$ & 0.0031 & $8.6646 \cdot 10^{-14}$ \\
\hline 2 & 0.0726 & 0.0600 & $1.2632 \cdot 10^{-15}$ & $3.5726 \cdot 10^{-13}$ \\
\hline 3 & 0.1686 & 0.0856 & $6.7289 \cdot 10^{-15}$ & 0.0036 \\
\hline 4 & 0.3701 & 0.3197 & $5.4318 \cdot 10^{-15}$ & $1.4743 \cdot 10^{-12}$ \\
\hline
\end{tabular}

TABLE IV: Parameter magnitude of the regularized model: weighted $\ell_{2,1}$-norm elements

\begin{tabular}{|c|c|c|c|c|}
\hline $\mathbf{i}$ & $\frac{\left\|\psi_{i}\right\|\left\|_{\infty}\right\| \theta_{A^{(i)}} \|_{2}}{\left\|\hat{\theta}_{A^{(0)}}\right\|_{2}}$ & $\frac{\left\|\psi_{i}\right\|_{\infty}\left\|\theta_{B^{(i)}}\right\|_{2}}{\left\|\hat{\theta}_{B^{(0)}}\right\|_{2}}$ & $\frac{\left\|\psi_{i}\right\|\left\|_{\infty}\right\| \theta_{C^{(i)}} \|_{2}}{\left\|\hat{\theta}_{C^{(0)}}\right\|_{2}}$ & $\frac{\left\|\psi_{i}\right\|_{\infty}\left\|\theta_{D^{(i)}}\right\|_{2}}{\left\|\hat{\theta}_{D^{(0)}}\right\|_{2}}$ \\
\hline 1 & 0.0242 & $8.2804 \cdot 10^{-15}$ & 0.0218 & $5.1653 \cdot 10^{-11}$ \\
\hline 2 & 0.0068 & 0.2331 & $3.5501 \cdot 10^{-15}$ & $8.5199 \cdot 10^{-11}$ \\
\hline 3 & 0.0063 & 0.1331 & $7.5653 \cdot 10^{-15}$ & 0.3447 \\
\hline 4 & 0.0055 & 0.1988 & $2.4431 \cdot 10^{-15}$ & $5.6270 \cdot 10^{-11}$ \\
\hline
\end{tabular}

1) Model sparsity: Tables III-IV show for the regularized model the magnitude of the parameters of the matrices linked to the different basis functions. The magnitude of the parameters in $B_{i}, \forall i=1, C_{i}, \forall i=2,3,4$ and $D_{i} \forall i=1,2,4$ of the regularized model is significantly smaller than the magnitude of the parameters in $B_{0}, C_{0}$ and $D_{0}$, respectively. Hence, the applied $\ell_{2,1}$-norm regularization enabled us to select a simpler scheduling parameter dependency, in the sense that the output equation is now modeled with six basis functions fewer than 


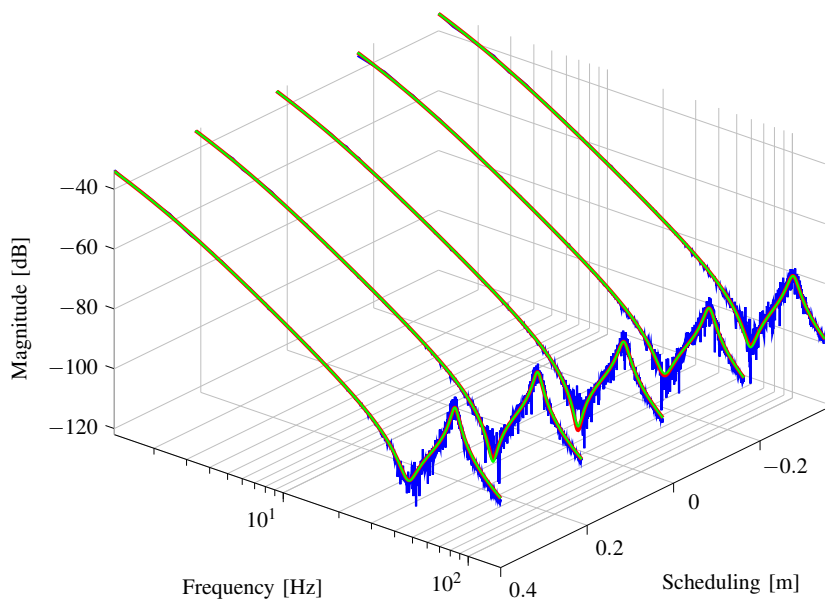

Fig. 3: Local identification fit of the first (rotary encoder) output - magnitude. Blue - measured FRFs, red - the SMILE model, green - the regularized model.

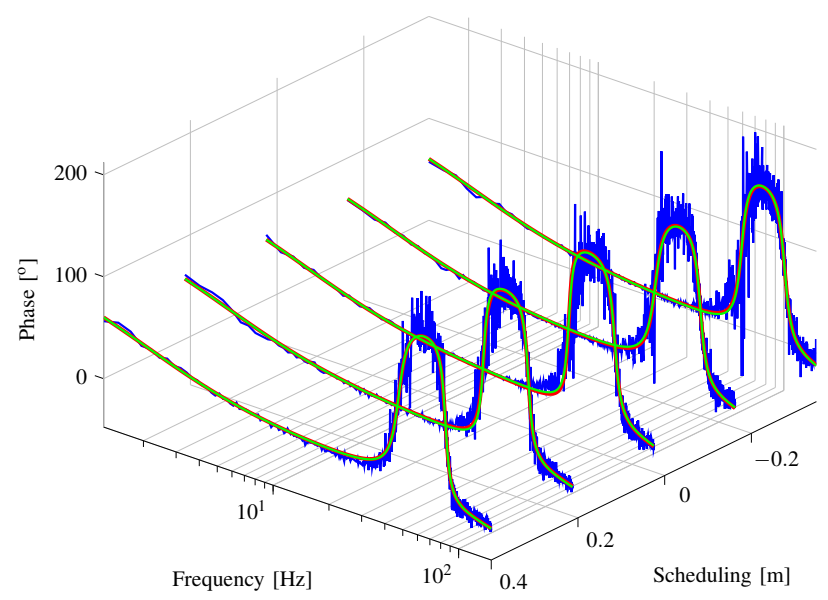

Fig. 4: Local identification fit of the first (rotary encoder) output - phase. Blue - measured FRFs, red - the SMILE model, green - the regularized model.

initially. Similarly, the $\mathscr{B}$ matrix is a basis function "lighter" than the initial input matrix.

2) Local validation: By looking at Figures 3-6, which portay the fit of the initial SMILE model and the regularized model-both with the integrator added back-to the measured FRFs, one can see no significant difference between the two. Their overlap with the measured FRFs evidently implies that both models capture the local LPV behavior of the ball screw sufficiently well. A very similar fit can be seen in Figures 7 8 , where the obtained model is evaluated at the position not used in the identification procedure, that is, at $p=-0.0688 \mathrm{~m}$, which proves that the model has satisfying interpolating capabilities. As mentioned in the experiment design (subsection III-A), the total sample variance is a measure of the nonlinear distortions, measurement and process noise. The fact that the model error magnitude is at the level of the estimated standard deviation of the measured FRFs $\sigma_{G_{u y_{i}}}, \forall i=1,2$ confirms that

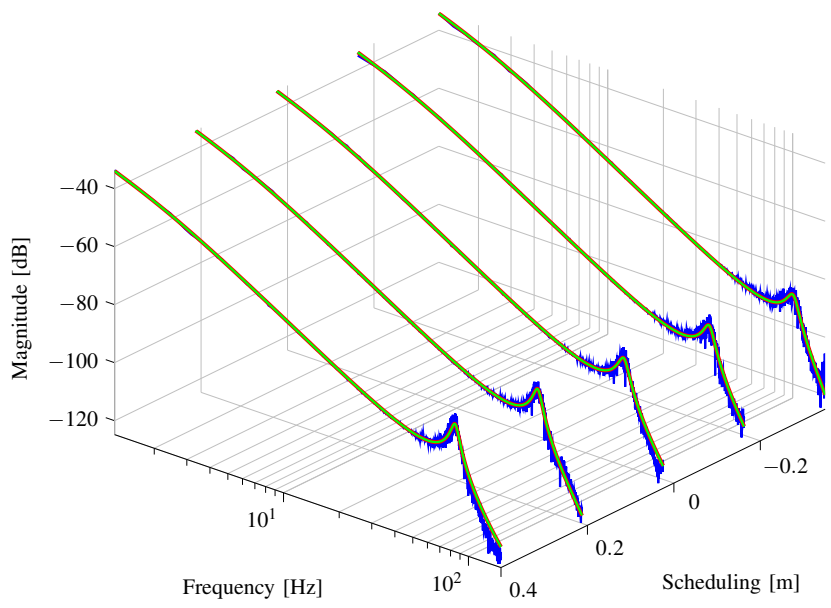

Fig. 5: Local identification fit of the second (linear encoder) output - magnitude. Blue - measured FRFs, red - the SMILE model, green - the regularized model.

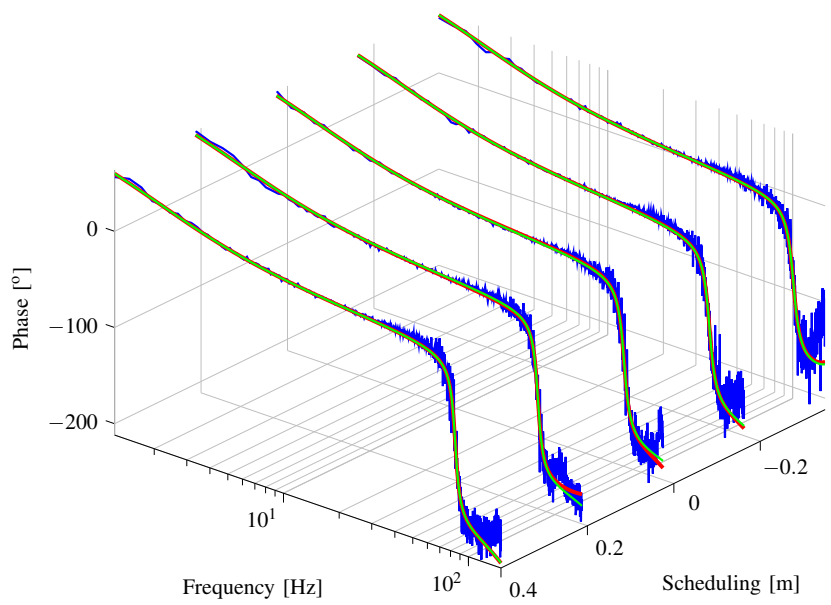

Fig. 6: Local identification fit of the second (linear encoder) output - phase. Blue - measured FRFs, red - the SMILE model, green - the regularized model.

the obtained accuracy is very close to the maximum one can achieve. Comparison of the estimates of the standard deviation of the FRFs, $\sigma_{G_{u y_{i}}}$, and of the noise, $\sigma_{n_{i}}$, reveals also that system nonlinearities are significant, especially around the system resonances.

3) Global validation: What remains is to check the model performance on global data, i.e. on data measured in an experiment where both the excitation signal and the scheduling parameter are continuously changing. A global experiment was performed in the closed-loop setting, with the referenceand hence the scheduling parameter-being a sine ensuring a smooth motion from one side of the ball screw to the other: $p(t)=r(t)=0.35 \sin (2 \pi 0.2442 t)$. The excitation signal $u_{\mathrm{ms}}$ is a random-phase multisine composed of frequencies in the range $f \in[10,150] \mathrm{Hz}$, focussing on the excitation of the system's resonance. Seven periods of the steady-state response were measured. Subsequently, the frequency content within 


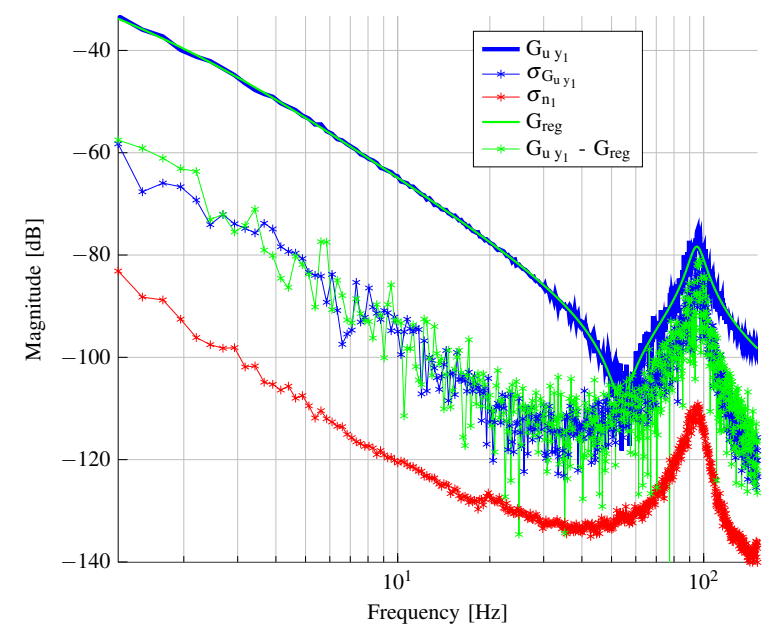

Fig. 7: Local validation fit for the first (rotary encoder) output - magnitude. $G_{u y_{1}}$ and $G_{\text {reg }}$ indicate the measured FRF and the FRF of the regularized model, respectively. $\sigma_{G_{u y_{1}}}$ indicates the estimated standard deviation of the FRF data and $\sigma_{n_{1}}$ estimated standard deviation of the noise.

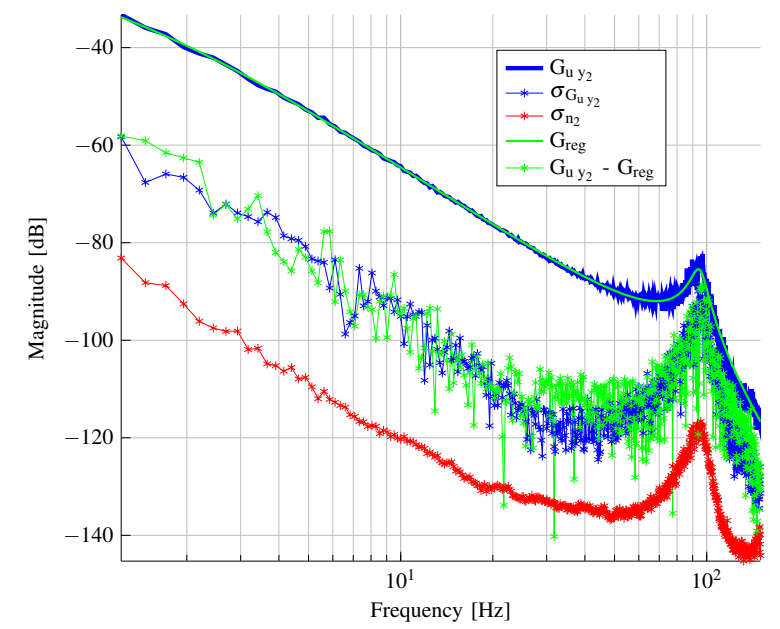

Fig. 8: Local validation fit for the second (linear encoder) output - magnitude. $G_{u y_{2}}$ and $G_{\text {reg }}$ indicate the measured FRF and the FRF of the regularized model, respectively. $\sigma_{G_{u y_{2}}}$ indicates the estimated standard deviation of the FRF data and $\sigma_{n_{2}}$ estimated standard deviation of the noise.

the multisine frequency range is extracted from the measured signals through frequency domain filtering (windowing). The resulting signals are compared with the results of a closedloop simulation of the LPV model performed with the same reference sine and multisine excitation. The same frequency domain filtering is applied to the resulting simulated outputs.

The difference between the measurements and simulation results is a measure of global accuracy of the LPV model. This difference contains a component that is systematic over the periods, resulting from systematic modeling errors, and a stochastic component resulting from process and measurement noise. Estimates of these two components can be obtained by calculating the average periodic error and substracting this average from the total error. The ratio of the standard deviation of the total error to its stochastic component equals 2.76, and 2.58 for output $y_{1}$ and $y_{2}$, respectively, indicating a large modeling error. To quantitatively judge this modeling error, additional frequency domain measurements were performed for one fixed reference position. The procedure for variance estimation described in section III-A was applied using four realisations of a random-phase multisine with a frequency content equal to that used in the global experiment. For each realisation, $N_{p}=7$ periods of the steady-state response were measured. The square root of the average (over the frequencies) ratio of the resulting total sample variance to the total noise variance for both outputs was calculated and equals:

$$
\sqrt{\left(\frac{\sigma_{G_{u y_{1}}}^{2}}{\sigma_{n_{1}}^{2} N_{p}}\right)}=2.90, \sqrt{\overline{\left(\frac{\sigma_{G_{u y_{2}}}^{2}}{\sigma_{n_{2}}^{2} N_{p}}\right)}}=2.58,
$$

where $N_{p}$ rescales the noise variance to account for the averaging. These two ratios confirm that the errors introduced by the nonlinearities are significantly larger than the noise contributions. Since they are almost the same as the ratios estimated from the global experiment, it can be concluded that the large systematic modeling errors revealed above are due to system nonlinearities rather than a systematic error in the LPV model.

\section{CONCLUSION}

This paper gives practical insights into the identification of an industrial ball screw setup by applying a nonlinear least-squares LPV system identification method with $\ell_{2,1}$-norm regularization. The identified state-space model is sparse. The magnitude of the frequency domain modeling error is at the level of the estimated standard deviation of the measured FRFs proving that the model captures the local LPV behavior of the ball screw sufficiently well. The model also shows reliable performance when used in conditions with continuously changing scheduling parameter. In future research, the resulting model will be used to design and validate robust $\mathscr{H}_{\infty}$ linear feedback controllers.

\section{REFERENCES}

[1] B. Henke, O. Sawodny, and R. Neumann, "Distributed parameter modeling of flexible ball screws using Ritz series discretization," IFAC Proceedings Volumes, vol. 47, no. 3, pp. 10132-10137, 2014.

[2] F. Felici, J.-W. Van Wingerden, and M. Verhaegen, "Subspace identification of MIMO LPV systems using a periodic scheduling sequence," Automatica, vol. 43, no. 10, pp. 1684-1697, 2007.

[3] J. De Caigny, R. Pintelon, J. Camino, and J. Swevers, "Interpolated modeling of LPV systems based on observability and controllability," in Proc. of the 16th IFAC Symposium on System Identification, Brussels, Belgium, pp. 1773-1778, 2013.

[4] D. Turk, J. Gillis, G. Pipeleers, and J. Swevers, "Identification of linear parameter-varying systems: A reweighted $\ell_{2,1}$-norm regularization approach," Mechanical Systems and Signal Processing, vol. 100, pp. 729 742, 2018, in press.

[5] E. J. Candes, M. B. Wakin, and S. P. Boyd, "Enhancing sparsity by reweighted $\ell_{1}$ minimization," Journal of Fourier analysis and applications, vol. 14, no. 5-6, pp. 877-905, 2008.

[6] R. Pintelon and J. Schoukens, System identification: a frequency domain approach, Second Edition. John Wiley \& Sons, 2012 